\title{
Corneal pseudocyst following acute hydrops
}

\author{
CURTIS E MARGO' AND MATTHEW W MOSTELLER 2
}

From the 'Department of Ophthalmology, University of South Florida College of Medicine, and the ${ }^{2}$ Eye, Ear, Nose and Throat Clinical Associates, Mobile, Alabama, USA

SUMmARY A 20-year-woman with bilateral keratoconus developed acute hydrops of her right cornea. The amount of corneal oedema progressively increased over eight weeks while the central cornea thinned. Histologically the cornea contained a stromal pseudocyst that was in continuity with the anterior chamber through breaks in Descemet's membrane. Pseudocyst formation is a rare complication of acute corneal hydrops that can simulate severe corneal ectasia.

Rapidly developing corneal oedema may complicate keratoconus when breaks in Descemet's membrane allow the uncontrolled influx of aqueous into stroma. Usually, however, acute hydrops resolves in several months as endothelial cells fill in the disrupted portion of posterior stroma and deposit new basement membrane. ${ }^{1-1}$ After healing, a residual stromal scar can result in decreased vision necessitating surgery. We have recently cared for a patient with keratoconus who developed a corneal pseudocyst following acute hydrops. The pseudocyst clinically simulated severe corneal ectasia.

\section{Case report}

A 20-year-old woman with classic bilateral keratoconus had been dependent on hard contact lenses for two years for clear vision. The patient had no history of connective tissue disease or other systemic diseases. Best corrected visual acuity had been $6 / 6$ in both eyes when she developed a painful right eye and blurred vision.

On examination one week after the onset of symptoms her vision was counting fingers, and there were a protruding $8 \mathrm{~mm}$ central corneal opacity and marked corneal oedema of the right eye. The anterior chamber could not be visualised. Best corrected visual acuity in the left eye was $6 / 6$, and the remainder of the ocular examination was normal.

The patient was treated with cycloplegic eye drops and pressure patching for the relief of pain. Over the next month the patient's visual acuity decreased to hand motion and her corneal oedema worsened and

Correspondence to Curtis E Margo, MD, Department of Ophthalmology, University of South Florida, Box 21, Tampa, FL 33612 . USA. spread centrifugally to the limbus. The cornea appeared to have thinned considerably. A penetrating keratoplasty was performed eight weeks after the onset of hydrops.

The corneal button was $2.9 \mathrm{~mm}$ thick centrally. A central stromal cyst, measuring more than $1 \mathrm{~mm}$ in diameter, drained clear fluid when the button was bisected. Light microscopy revealed severe epithelial and stromal oedema, disordered keratocyte proliferation, and multiple breaks in Bowman's membrane. The stromal cyst was partially lined by several layers of proliferating keratocytes (Fig. 1). Several breaks in Descemet's membrane were present (Fig. 2). Thin channels running at oblique angles from the cyst through posterior stroma to breaks in Descemet's membrane were demonstrated on serially sectioning the cornea and verified the continuity of the cyst with the anterior chamber.

Following surgery the patient was treated with tapering doses of a cycloplegic and topical dexamethasone. The patient's uncorrected visual acuity three months after surgery was $6 / 21$.

\section{Discussion}

Pseudocysts are acquired fluid filled cavities lined by fibroconnective tissue that differ from true cysts because they lack an epithelial lining. ${ }^{5}$ They may form after a variety of injuries throughout the body when failing homoeostatic mechanisms cannot prevent fluid from accumulating in the extracellular space. ${ }^{56}$

Corneal oedema resulting from breaks in Descemet's membrane usually resolves with time. ${ }^{2-1}$ In this case, however, the extent and amount of oedema progressively increased. Pseudocyst forma- 
Fig. 1 A large cystic space (CY) is present in the central cornea. Several layers of keratocytes (arrows) line a portion of the cavity. Small interruptions in Descemet's membrane posterior to the cyst are artefact. Haematoxylin-eosin.

Fig. 2 An extension of the stromal cyst (CY) could be traced posteriorly to a break in Descemet's membrane (arrows). No endothelial cells are present in this section. Haematoxylin-eosin.
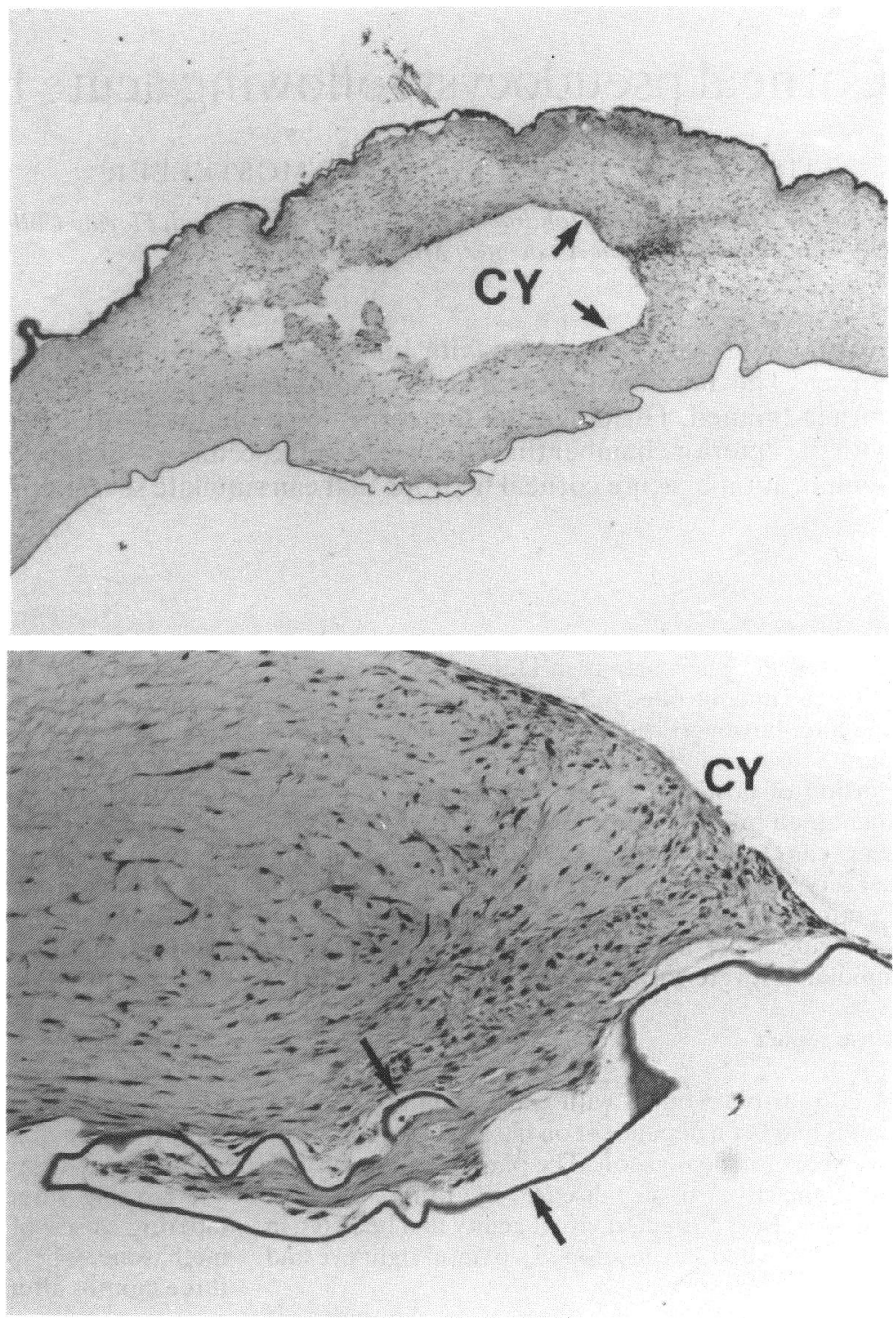

tion was the result of uncontrolled influx of aqueous into the corneal stroma through rents in Descemet's membrane. Since the pseudocyst in our patient could not be delineated through the opacified stroma, the stroma anterior to the cyst was mistakenly interpreted as being the total thickness of a severely ectatic cornea. A review of keratoconus and of related non-inflammatory corneal thinning disorders did not describe pseudocyst formation as a complication of hydrops. ${ }^{4}$ We are unaware of any previous reports describing the occurrence of pseudocyst formation in this clinical setting.

\section{References}

1 Chi H, Katzin H, Teng C. Histopathology of keratoconus. Am J Ophthalmol 1956; 42: 847-60.

2 Stone DL, Kenyon KR, Stark WJ. Ultrastructure of keratoconus with healed hydrops. Am J Ophthalmol 1976; 82: 450-8.

3 Wolter JR, Henderson JW, Clahassey EG. Rupture of Descemet's membrane in keratoconus causing acute hydrops and posterior keratoconus. Am J Ophthalmol 1967; 63: 1689-92.

4 Krachmer JH, Feder RS, Belin MW. Keratoconus and related non inflammatory corncal thinning disorders. Surv Ophthalmol 1984; 28: 293-322.

5 Rosen VJ. The pancreas. In: Coulson WF, ed. Surgical pathology. Philadelpha: Lippincott, 1978: 280.

6 Waugh JM, Lynn TE. Clinical and surgical aspects of pscudocyst: analysis of 58 cases. Arch Surg 1958; 77: 47-54.

Accepted for publication 23 July 1986. 